\title{
Two new techniques for obtaining consistent results when growing Renibacterium salmoninarum on KDM2 culture medium
}

\author{
T. P. T. Evelyn, L. Prosperi-Porta, J. E. Ketcheson \\ Department of Fisheries and Oceans, Biological Sciences Branch, Pacific Biological Station, Nanaimo, British Columbia, \\ Canada V9R $5 \mathrm{~K} 6$
}

\begin{abstract}
We recently showed that the inconsistent performance of the culture medium KDM2 in supporting growth of the fastidious kidney disease agent (Renibacterium salmoninarum = Rs) was due to a lot-to-lot variation in the composition of a commercially available peptone used as a medium ingredient. We now report on 2 procedures for obtaining consistent growth of the pathogen on KDM2, regardless of the lot of peptone used in preparing the medium. The first procedure involves the recently described nurse culture technique that has the added advantages of accelerating growth of Rs and of increasing the sensitivity with which the pathogen can be detected. The second procedure is to supplement KDM2 with a small amount of KDM2 broth that has previously been used to grow Rs (= spent broth). A metabolite (or metabolites) produced by the pathogen during its growth in the broth appears to substitute effectively for the nurse culture by providing the Rs growth initiating factor(s) that, apparently, is in short supply in certain lots of peptone. These techniques remove the need to pre-test peptone lots to ensure that they will perform satisfactorily when used in KDM2 for growing Rs.
\end{abstract}

\section{INTRODUCTION}

Renibacterium salmoninarum (Rs), the causative agent of bacterial kidney disease (BKD) in salmonids, is a fastidious and slow-growing organism. Recently, Evelyn et al. (1989) described a technique, the nurse culture technique, that significantly accelerates the growth of the pathogen when the organism is present in low numbers. The technique takes advantage of the fact that low numbers of Rs cells always show accelerated growth when they are plated on an agar medium (KDM2) adjacent to a nurse culture consisting of a heavy inoculum of Rs cells. Until recently, it was thought that growth acceleration of Rs and an increased sensitivity at detecting the pathogen were the only benefits of the technique. The experiments outlined below, however, highlight another advantage of the nurse culture technique. We have reported frustrating instances in which the normally satisfactory Rs culture medium, KDM2, failed to support growth of the pathogen (Evelyn \& Prosperi-Porta 1989). An investigation of these occurrences revealed that they were due to the particular production lot of peptone used in preparing the culture medium. Production lots of pep- tone varied enormously in their growth promoting properties for Rs even when they were produced by the same manufacturer. The purpose of this paper is to show that growth failures of Rs on KDM2 due to the inadvertent use of faulty lots of peptone can be avoided by (1) using the nurse culture technique or (2) supplementing the growth medium with an unidentified metabolite(s) produced by the nurse culture.

\section{MATERIALS AND METHODS}

General. Two experiments were performed. The first examined the effect of the nurse culture technique on the growth of Rs resulting on batches of KDM2 prepared with production lots of peptone that had previously been shown to be satisfactory or unsatisfactory at promoting growth of Rs (Evelyn \& Prosperi-Porta 1989). In the second experiment, Rs was grown from a heavy inoculum in KDM2 broth and the spent broth was tested to determine whether it contained Rs metabolites that would substitute for the nurse culture in KDM2 prepared with an unsatisfactory lot of peptone. The 3 Rs isolates used in the experiments (Rs 384, Rs 
8894, Rs 9902) were obtained from different stocks of cultured chinook salmon Oncorhynchus tshawytscha, affected with BKD. Each isolate was typical of Rs and 2 of them have been described elsewhere (Evelyn et al. 1989). The Rs cultures were 8 to 16 d old when used in the experiments. The 4 lots of peptone used for preparing the various batches of KDM2 had earlier been shown to be satisfactory (Lot 4) or unsatisfactory (Lots 2, 3, and 7) for promoting the growth of Rs (Evelyn \& Prosperi-Porta 1989, Evelyn et al. unpubl.).

In the first experiment, 3 different batches of KDM2 (Evelyn \& Prosperi-Porta 1989) were prepared, each with a different production lot of peptone (Lot 2, 3, or 4). Petri plates, poured with a particular batch of KDM2, were then drop-inoculated (Miles \& Misra 1938) peripherally with $25 \mu \mathrm{l}$ aliquots of Rs cells suspended in peptone $(0.1 \%)$ saline $(0.85 \% \mathrm{NaCl})$ (= PS). The inocula consisted of cells of Rs 8894 or Rs 9902 that were suspended in PS to a turbidity of 1.25 OD at 540 $\mathrm{nm}$ and then diluted to $10^{-3}, 10^{-4}$, and $10^{-5}$. A single dilution of a single Rs isolate was inoculated onto any given plate. Those plates inoculated peripherally with Rs 8894 were simultaneously inoculated centrally with a $25 \mu l$ drop of nurse culture (Rs 9902 cells in PS at 1.25 $O D$ at $540 \mathrm{~nm}$ ) or with a $25 \mu \mathrm{l}$ drop of sterile PS (controls). Plates inoculated peripherally with Rs 9902 were simultaneously inoculated centrally with a $25 \mu \mathrm{l}$ drop of nurse culture (Rs 384 cells in PS at 1.25 OD at $540 \mathrm{~nm}$ ) or with a $25 \mu \mathrm{l}$ drop of sterile PS (controls). Following imbibition of the drops of inoculum, plates were sealed in plastic bags to prevent desiccation, and incubated inverted, at $15^{\circ} \mathrm{C}$ for 20 or $21 \mathrm{~d}$. At this stage, counts of colonies developing from each $25 \mu$ drop of $10^{-5}$-diluted suspension were made using a stereomicroscope at a magnification of 120 . In addition, the turbidity yielded by the growth from the drops of the variously diluted suspensions was measured at $540 \mathrm{~nm}$, using the procedure outlined earlier (Evelyn et al. 1989)

In Expt 2, 2 batches of KDM2 were prepared, one with a satisfactory lot of peptone (Lot 4 ), the other with an unsatisfactory lot of peptone (Lot 7). Petri plates, poured with these production lots of peptone (1 batch of KDM2 plate ${ }^{-1}$ ), were drop-inoculated peripherally with Rs 384 cells ( $25 \mu$ d drops of cells suspended in PS to a turbidity of $1.25 \mathrm{OD}$ at $540 \mathrm{~nm}$ and diluted to $10^{-3}$. $10^{-4}$, and $10^{-5}$ ). These plates were not inoculated with nurse cultures. Instead, they were inoculated' centrally with spent, sterile, $10 \times$ concentrated KDM2 broth, the preparation of which is described below, or with fresh (unspent), sterile, $10 \times$ concentrated KDM2 broth (controls). Spent KDM2 broth was prepared as follows. Several loopfuls of Rs 384 cells from a KDM2 plate were inoculated into a flask containing $100 \mathrm{ml}$ of KDM2 broth (prepared with peptone Lot 4) and the culture was incubated at $15^{\circ} \mathrm{C}$ with occasional shaking until a turbidity of $0.83 \mathrm{OD}$ at $540 \mathrm{~nm}$ was reached $(18 \mathrm{~d}$ ). At this point, the culture was centrifuged to remove the Rs cells and other particulate matter $(12000 \times g$ for $30 \mathrm{~min}$ at $4{ }^{\circ} \mathrm{C}$ ). The clarified supernatant was then freezedried and redissolved in $1 / 10$ th of its original volume using distilled water. The concentrated supernatant was then filter-sterilized directly, using a $0.22 \mu \mathrm{m}$ pore diameter filter (Millipore), or following its passage through a molecular sieve (nominal molecular weight limit of 10000 Daltons, Millipore). In addition, sieved material was also sterilized by autoclaving $\left(121^{\circ} \mathrm{C}\right.$ for $15 \mathrm{~min}$ ). Samples (25 $\mu$ ) ) of the variously treated spent broth concentrate were applied centrally (in place of the nurse culture) on test plates of KDM2 (1 sample plate $\left.{ }^{-1}\right)$. Control plates were 'inoculated' centrally with $25 \mu \mathrm{l}$ of sterile, $10 \times$ concentrated, unspent KDM2 broth. Plates were then incubated, as described above, for $19 \mathrm{~d}$, at which time the resulting Rs colonies were recorded turbidometrically $\left(10^{-3}\right.$ sample) and photographically $\left(10^{-4}\right.$ sample $)$, or counted $\left(10^{-5}\right.$ sample).

\section{RESULTS AND DISCUSSION}

Results indicate that when the nurse culture technique was used, growth of Rs was consistently obtained on all batches of KDM2, irrespective of the lot number of the peptone used to prepare the medium. In contrast, when nurse cultures were not used, only that batch of KDM2 prepared with a peptone lot (Lot 4), previously shown to promote satisfactory growth of Rs, supported growth of Rs. These results held true for each of the 2 Rs isolates tested (Table 1), indicating that the nurse culture phenomenon is not specific to a particular isolate. Clearly, reproductible results in growing Rs depend on using batches of KDM2 prepared with previously proven peptone lots or on using the nurse culture technique. The latter approach, however, provides 2 additional advantages: faster Rs growth and increased sensitivity in detecting Rs cells (Evelyn et al. 1989). These advantages were again obvious with the 2 Rs isolates in the present experiment when their short term ( 20 or 21 d) growth, obtained from the more highly diluted samples (the $10^{-4}$ and $10^{-5}$ diluted samples), was considered. In all cases, there was no detectable growth on any of the batches of KDM2 media - not even on the batch of KDM2 prepared with the satisfactory lot (Lot 4) of peptone - when the nurse culture technique was not used. In contrast, when nursed, both isolates grew well on all batches of KDM2 even at these dilutions of inoculum (turbidities on the various batches of medium inoculated with the highest dilution of inoculum averaged, for example, 0.100 OD for Rs 8894 and 0.096 OD for Rs 9902). 
